\title{
Efectos de dos métodos de entrenamiento de fuerza sobre el Índice de Bosco en jugadoras de balonmano deDivisión deHonor Effects of two strength training methods in Bosco Index on elite handball female player
}

\author{
María del Carmen Ferrer Contreras \\ Universidad deAlmería
}

\begin{abstract}
Resumen: SEl presente trabajo es un estudio de ámbito deportivo, donde se pretende conocer y mejorar la manifestación de fuerza menos desarrollada, modificando el Índice de Bosco, utilizando dos programas de entrenamiento de la fuerza por contraste acentuado en la serie. Para ello, se ha seleccionado una muestra de nueve jugadoras de división de honor con edades comprendidas entre los 18 y 32 años siendo divididas en dos grupos. Un grupo formado por aquellas jugadoras que han obtenido en el pretest unos valores más bajos en el Índice de Bosco, que llevará a cabo un programa de entrenamiento de fuerza por contraste acentuado en la serie con cargas máximas y pliometría y el otro grupo por las que consiguieron unos valores más altos, que realizarán un programa de entrenamiento de fuerza por contraste acentuado en la serie con cargas medias y pliometría, a los cuales serán sometidas durante un periodo de diez semanas, ejecutando dos sesiones en cada una. Los resultados obtenidos de la investigación muestran diferencias significativas en el pretest entre ambos grupos, lográndose igualar los Índices de Bosco de ambos grupos en el test intermedio y postest.
\end{abstract}

Palabras clave: Test de Bosco. Entrenamiento. Balonmano. Pliometría. Índice de Bosco.

\begin{abstract}
The current work is a sports field study, where the purpose is to know and to improve the less develop strength display, altering the Bosco Index, using two strength training programs by series stressed contrast. For that, it has been choosing a sample of nine elite female players between 18 and 32 years old, being splat in two groups up. One group was set for those players whose got the lowest Bosco Index scores, and made a strength training program with maximum loads and plyometric. The other group was set with the players whose got the highest Bosco Index scores, and did the strength training program with medium loads and plyometric. Both groups were training during ten weeks, twice a week. The research outcomes show significant differences in both groups during pretest, achieving to make equal the Index Bosco during the intermediate test and post test.
\end{abstract}

Key words: Bosco Test. Training. Handball. Plyometric. Strength.

\section{Introducción.}

La sociedad actual, está transformando el modelo deportivo, haciendo que la manifestación más habitual del deporte, la competitiva, pase en estos momentos a un segundo plano, sin embargo, los padres siguen acercando casi exclusivamente a sus hijos/as a la iniciación deportiva y la competición (Nuviala et al., 2003). El balonmano es un deporte, o una forma de trabajo como es el caso de los deportistas profesionales (Chillón et al., 2002), que requiere tener unas buenas cualidades físicas para su práctica. La fuerza y la velocidad, son las que más imperan, siendo necesarias para la ejecución de diferentes gestos deportivos (Lara et al., 2005). La capacidad de salto es una de las cualidades más importantes en este deporte, ya que se utiliza en muchos gestos, tanto en ataque como en defensa (lanzamientos, bloqueos, superar barreras,...). Por estos motivos es un gesto básico para los practicantes de balonmano, debiendo ocupar un puesto importante entre las rutinas de entrenamiento de dichos deportistas (Antón, 1991), considerando que las acciones de juego que se suceden, requieren de una gran fuerza aplicada en el menor tiempo posible, lo que se conoce como manifestación de fuerza explosiva (Vittori, 1990).

Poder evaluar la fuerza y velocidad del músculo en todos sus aspectos y expresiones, ha sido una tarea difícil hasta que Bosco (1986) citado por Bosco (1994), propuso como solución la confrontación de la capacidad de salto (que puede representar perfectamente la máxima expresión de la velocidad de contracción de los elementos contráctiles de los músculos extensores de las piernas en condiciones naturales) con la actividad muscular ejercida cuando se debe levantar una carga similar al peso corporal del sujeto.

Según Bosco (1994), la valoración de la fuerza máxima y la máxima velocidad se pueden registrar en una misma unidad de

Fecha de recepción: 07-11-06 - Fecha de aceptación: 20-01-07 Correspondencia: María del Carmen Ferrer Contreras

$\mathrm{C} /$ Manuel Azaña, $131,3^{\circ} \mathrm{C}$

04006 Almería

E-mail: maricarmenferrer@yahoo.es medida, que puede ser la altura alcanzada o la velocidad vertical de despegue de la que depende la altura, siendo esto lo que nos va a permitir establecer una relación entre la fuerza y velocidad, en un músculo aislado o en un grupo muscular. Del equilibrio entre la fuerza y la velocidad se obtiene el denominado Índice de Bosco (IB), un valor numérico que relaciona la capacidad de salto del atleta cuando tiene una carga sobre los hombros y cuando efectúa el salto sin sobrecarga.

De esta forma se puede evaluar las características de los sujetos o si los resultados obtenidos después de un entrenamiento han desarrollado homogéneamente el músculo o impera una propiedad con respecto a otra (más velocidad que fuerza o más fuerza que velocidad), lo que podría producir un desequilibrio dentro del músculo.

Si el IB es muy alto o crece con el entrenamiento, significa que se está dando mayor importancia al trabajo de fuerza máxima o al menos así se manifiesta en el sujeto entrenado; por el contrario, si decrece o es muy bajo posiblemente se estará primando el trabajo de velocidad (González y Gorostiaga, 1995).

Para Cometti (2001) es preciso utilizar la alternancia entre cargas y así evitar la adaptación que impide el desarrollo de la fuerza. De esta forma surge el método de Contraste, en el que se alternan cargas pesadas con cargas ligeras en una misma sesión de entrenamiento. Esta alternancia, no sólo es eficaz cuando se mezclan cargas de diferentes intensidades, sino que también lo son cuando se combinan distintos tipos de contracciones (Cometti, 1988). En esta línea y siguiendo a García et al. (1996), se indica que el método de contraste tiene un objetivo mixto de mejora de la fuerza máxima y de la fuerza veloz, que consiste en alternar cargas altas (70-90\%) con ligeras (30-50\%).

Dentro del método de Contraste, la forma más conocida es el método Búlgaro y sus diferentes variantes a la hora de ejecutar los ejercicios, en la composición interna de las series o en la ejecución de la sesión. En su origen, el método Búlgaro solo consideraba los ejercicios con cargas, pero este método según García et al. (1996, p. 199) «es aplicable igualmente a la alternancia de ejercicios o movimientos en los que no necesariamente se utilicen cargas» (p. e., el trabajo pliométrico), especialmente 
cuando está dedicado a disciplinas con carácter explosivo, como es el caso del deporte del balonmano.

Para Cometti (2001), la acentuación del efecto del contraste es la que produce los efectos positivos de la alternancia de los ejercicios con cargas y sin cargas (gran tensión sobre carga pesada-velocidad de ejecución con el propio peso del cuerpo).

Siguiendo a Rodríguez y García (1998), tanto el método de entrenamiento por contraste como el método pliométrico, producen incrementos en la fuerza de los sujetos, mejorando por consiguiente la altura del salto. En cambio, aunque los métodos pliométricos producen resultados favorables sobre la fuerza explosiva, no se ha comprobado si éstos son mejores combinándolos con otros métodos. Chirosa (1998), manifiesta que el método de contraste se podría utilizar combinando la pliometría con métodos concéntricos, isométricos y excéntricos, logrando mejorar los gestos explosivos.

En esta línea argumental, el objetivo de este trabajo es mejorar la manifestación de la fuerza menos desarrollada a través del método búlgaro por contraste acentuado (con cargas máximaspliometría y cargas medias-pliometría) medida por medio del IB.

\section{Material y método.}

\subsection{Sujetos.}

Se han elegido como sujetos experimentales a 13 jugadoras de balonmano de división de honor femenina, pertenecientes al Club de Balonmano Vícar Goya Jarquil de Almería. Las lesiones sufridas en el transcurso del estudio impidieron a algunas jugadoras poder realizar los entrenamientos con normalidad, produciéndose 4 muertes experimentales, quedando el grupo reducido a 9 jugadoras. La edad de los sujetos oscilaba entre los 18 y los 32 años, la talla entre 159.0 y 183.5 centímetros y el peso de 54.0 a 80.0 kilogramos.

Además de controlarles estas variables, se consideró interesante tener en cuenta los años de entrenamiento, no estar lesionada, no tener una enfermedad que pudiera afectar al resultado de la prueba y no haber tomado, ni tomar medicamentos u otras sustancias que puedan afectar al desarrollo de la fuerza. Junto a todo lo anterior, se comprobó que pudiesen ejecutar media sentadilla con una carga del $100 \%$ de su peso corporal y que tuvieran unas condiciones de vida prácticamente idénticas.

Los sujetos se distribuyeron según las marcas conseguidas en el pretest, concretamente según el valor del IB, conformando el grupo de método búlgaro acentuado con carga máxima (GCAMA), aquellas que lograron unos niveles más bajos (entre 0.175 y 0.202$)$ y, por otro lado, el grupo de método búlgaro acentuado con carga media (GCAME), compuesto por las jugadoras que obtuvieron los índices más elevados (entre 0.215 y 0.312).

\subsection{Procedimiento.}

El procedimiento que se siguió, una vez que los sujetos fueron informados de su participación en esta investigación, consis- tió en la realización de un test inicial en el que se realizaron las siguientes pruebas: test de fuerza máxima en media sentadilla, para calcular el porcentaje de fuerza máxima, que era modificado cada dos semanas aumentando un 5\% la intensidad de la carga con respecto al porcentaje de la fuerza máxima inicial en media sentadilla de cada jugadora y así evitar la adaptación de los sujetos al estímulo, siguiendo uno de los principios del entrenamiento (principio de la progresión), según García et al. (1996), y test de Bosco.

Una vez realizados los tests se procedió a analizar el valor del IB clasificando los sujetos en dos grupos (GCAME y GCAMA) y de esta forma diseñar las sesiones de entrenamiento. A continuación, se aplicaron los entrenamientos de contraste durante dos días a la semana alternos (lunes y miércoles), en un periodo de diez semanas trabajando durante ese periodo el GCAMA con una carga que osciló entre el 80-90\% y el GCAME con el 55-65\%. En nuestro caso la combinación que se utilizó fue la pliometría con un método concéntrico, en concreto, el método Búlgaro por contraste acentuado en la serie, ya que según Chirosa (1998), es más eficaz.

Ambos grupos tuvieron que realizar el contraste de cada serie con saltos pliométricos de tipo Drop Jump (DJ), que consistía en efectuar un salto después de una caída de una altura determinada, partiendo de una posición de piernas extendidas y con un movimiento hacia abajo, debiendo llevar las manos en las caderas y el tronco recto y según la altura óptima de caída correspondiente a cada una de las jugadoras, siendo el número de saltos igual en los dos grupos.

Tras seis semanas de entrenamiento (12 sesiones), o lo que es lo mismo, ocho semanas desde que tuvo comienzo la investigación, se les realizó un test intermedio con las mismas pruebas que el pretest, para analizar la evolución de la forma y modificar la intensidad de las cargas, ajustándolas a la variación de fuerza producida por el entrenamiento. Se continuó con otras cuatro semanas más de entrenamiento (8 sesiones) y a la décimo quinta semana del comienzo de la investigación, se realizó la batería de test final (postest), consistente en la realización del test de Bosco que sirvió para medir la evolución del rendimiento, y su efecto sobre la variable dependiente. En la tabla 1 puede observarse, a modo de síntesis, los pasos seguidos en la aplicación del diseño de la investigación.

Las pruebas de las que estaba compuesto cada test, se realizaron en el Centro Andaluz de Medicina del Deporte de Almería (C.A.M.D.) por el Médico Deportivo bajo la observación de la investigadora principal y las sesiones de entrenamiento en un gimnasio de la localidad. Antes de realizarles la batería de pruebas que configuran el test de Bosco, todas las jugadoras fueron pesadas y medidas en el mismo centro en el que se hizo el test, siendo el peso un dato necesario para poder realizar una de las pruebas del test (SJ100\%).

\subsection{Aparataje.}

Para la aplicación del test de repeticiones máximas en media sentadilla, así como el test de Bosco se hizo necesario un aparataje

\begin{tabular}{|c|c|c|c|c|c|}
\hline \multicolumn{7}{|c|}{ Tabla 1. Pasos seguidos en la aplicación del diseño de investigación } \\
\hline \multirow{3}{*}{ Pretest } & $\begin{array}{c}\text { Asignación } \\
\text { sujetos a cada } \\
\text { grupo }\end{array}$ & $\begin{array}{c}\text { Tratamiento } \\
\left(1^{\mathrm{a}} \text { parte }\right) \\
(6 \text { semanas })\end{array}$ & $\begin{array}{c}\text { Test } \\
\text { intermedio }\end{array}$ & $\begin{array}{c}\text { Tratamiento } \\
\left(2^{\mathrm{a}} \text { parte }\right) \\
(4 \text { semanas })\end{array}$ & Postest \\
\hline \multirow{3}{*}{$\begin{array}{c}\text { Realización de } \\
\text { batería de tests }\end{array}$} & $\begin{array}{c}\text { Mayor I.B. } \\
\text { GCME }\end{array}$ & $\begin{array}{c}\text { Entrenamiento } \\
\text { fuerza-velocidad }\end{array}$ & $\begin{array}{c}\text { Realización de } \\
\text { batería de tests }\end{array}$ & $\begin{array}{c}\text { Entrenamiento } \\
\text { fuerza-velocidad }\end{array}$ & $\begin{array}{c}\text { Realización de } \\
\text { batería de tests }\end{array}$ \\
\cline { 2 - 7 } & $\begin{array}{c}\text { Genor I.B. } \\
\text { GCMA }\end{array}$ & $\begin{array}{c}\text { Entrenamiento } \\
\text { fuerza máxima }\end{array}$ & $\begin{array}{c}\text { Realización de } \\
\text { batería de tests }\end{array}$ & $\begin{array}{c}\text { Entrenamiento } \\
\text { fuerza máxima }\end{array}$ & $\begin{array}{c}\text { Realización de } \\
\text { batería de tests }\end{array}$ \\
\hline
\end{tabular}


que hizo posible la obtención de los datos, en concreto para la realización del test de Bosco se empleó el Ergo Tester Globus Italia que realiza las funciones de una plataforma de salto y ha sido utilizado en diferentes estudios como el de Mouche (2001). Para el test de fuerza máxima en media sentadilla fueron necesarios barras y discos de halterofilia de diferentes pesos y soportes. También se utilizaron otros instrumentos como una báscula con una precisión de 100 gramos y un tallímetro para medir la estatura de las personas con una precisión de 1 milímetro.

\subsection{Protocolo.}

El protocolo que se llevó a cabo para medir la variable dependiente consistió en un calentamiento que constaba de movilidad de las principales articulaciones del cuerpo, haciendo principal hincapié en el tren inferior, diez minutos de carrera continua, cinco minutos de estiramientos, diez saltos sobre las puntas y varios saltos para repasar la técnica.

El Test de cargas progresivas $\left(\mathrm{SJ}_{0}-\mathrm{SJ}_{50}\right.$ y $\left.\mathrm{SJ}_{100}\right)$, que se utiliza para valorar la manifestación de la fuerza máxima dinámica y la capacidad contráctil, está relacionado con la variable dependiente de nuestra investigación (Índice de fuerza velocidad o IB), y se trata de realizar el Squat Jump con cargas crecientes en función del peso corporal del deportista. El protocolo que se sigue es:

$\varnothing$ Posición inicial: la posición de partida es idéntica a la del SJ, es decir el sujeto se coloca de pie con una posición de semiflexión de rodillas $\left(90^{\circ}\right)$, pies paralelos, o con las puntas ligeramente hacia fuera y una separación similar a la anchura de las caderas, con la salvedad de que el movimiento debe efectuarse con las manos soportando una carga apoyada sobre los hombros y el tronco recto.

$\varnothing$ Ejecución: el movimiento es el mismo que el SJ o salto sin contramovimiento, tratando igualmente que el salto sea máximo. Consistía en la realización de un salto vertical máximo, partiendo de la posición de flexión de piernas de $90^{\circ}$ (medidos por un goniómetro manual), sin ningún tipo de rebote o contramovimiento. Los miembros superiores no intervienen en el salto ya que las manos sujetan la haltera. En la fase de vuelo, el sujeto debe mantener el cuerpo erguido, las piernas extendidas y pies en flexión plantar efectuando la caída en el mismo lugar de inicio Se descansa aproximadamente un minuto y lo repite, normalmente se suelen ejecutar dos intentos cogiendo el mejor de ellos.

Para poder ejecutar este salto, es necesario contar con una barra de halterofilia con sus correspondientes discos (hasta completar el peso del sujeto). Para este caso concreto se ha realizado con cargas del 25-50-75 y 100\% aunque sólo se ha considerado el valor del $\mathrm{SJ}_{100}$, necesario para obtener el valor del índice de Bosco, sirviendo los saltos con el resto de cargas para preparar la musculatura. Una vez realizado el salto con una carga correspondiente al $25 \%$ de su peso corporal, se hace el siguiente con el mismo protocolo y así sucesivamente hasta completar el test.

\subsection{A nálisis estadístico.}

Una vez finalizado el trabajo de campo, según el proceso de trabajo de esta investigación y los instrumentos utilizados, se ha realizado un análisis cuantitativo, descriptivo e inferencial. Para realizar este análisis se ha utilizado el paquete de programas informático SPSS (Statistical Package for the Social Sciences) para Windows, versión 12.0, que nos permite poner en práctica las técnicas estadísticas.

Primeramente se ha llevado a cabo un análisis descriptivo de las variable dependiente tanto a nivel general como de forma específica de cada uno de los grupos y a continuación se ha realizado un análisis inferencial particularizando en cada uno de los grupos que intervinieron en la investigación.

\section{R esultados.}

Aunque el I.B. fue calculado a trece sujetos experimentales inicialmente, siendo este el criterio con el que se clasificaron a los sujetos en dos grupos experimentales, sólo han podido ser analizados los valores de nueve sujetos que fueron los que continuaron todo el proceso de investigación. El mínimo alcanzado fue 0.12 (GCAMA) y el máximo 0.35 (GCAME), correspon-diendo al pretest y postest respectivamente (tabla 2). Esta variable evoluciona progresivamente en cada una de las mediciones como

\begin{tabular}{|l|l|l|l|l|l|}
\hline \multicolumn{5}{|c|}{} & \\
\hline & & & & & \\
\hline & & & & & \\
\hline & & & & & \\
\hline & & & & & \\
\hline
\end{tabular}

podemos observar en los diagramas de Box Whisker de la figura 1, elevándose la media de 0.22 a 0.29 , si bien para GCAMA, disminuye la dispersión, mientras que para el otro grupo (GCAME), se observa todo lo contrario, es decir, un aumento en la dispersión de los datos y en la desviación típica.

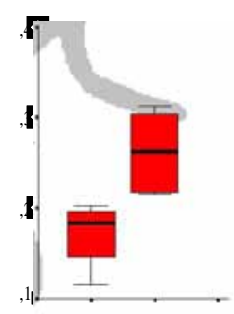

GCAMA GCAME

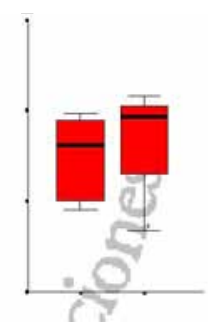

GCAMA GCAME

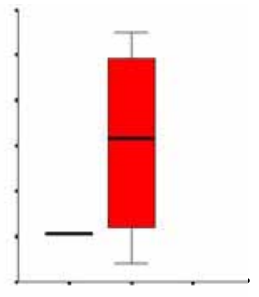

GCAMA GCAME

Postest
Figura 1. Diagrama de Box-Whisker para IB

Con respecto al análisis descriptivo de la variable dependiente en función del programa de tratamiento se utilizará la media, desviación típica y el número de sujetos en cada uno de los grupos y momento de medición (tabla 3 ).

Los valores de $\mathrm{N}$ (número de sujetos) para el pretest como para el test intermedio son de 9 pero hay que matizar que para el GCAMA en el postest es de 4, y de 5 en el test intermedio, ocurriendo lo contrario para el GCAME. Si nos fijamos en el postest, hay una pérdida de 4 sujetos experimentales, siendo $\mathrm{N}$ igual a 1 para el GCAMA y 4 para el GCAME. La media del IB en el pretest es de 0.09 superior para el GCAME con respecto al GCAMA acortándose la diferencia que existía y pasando a ser de 0.02. en el test intermedio y por último de 0.04 en el postest.

En el análisis inferencial, los resultados de los contrastes multivariados se han llevado a cabo entre tests (factor intrasujetos) y entre grupos (factor inter-sujetos). Comenzando con el factor intra-sujetos, los resultados muestran el p-valor asociado al estadístico Lambda de Wilks (0.224), indicando que no existen diferencias significativas entre los tests.

\begin{tabular}{|c|l|c|c|c|}
\hline \multicolumn{5}{|c|}{ Tabla 3. Prueba T, contrastes entre grupos en el pretest, test intermedio } \\
\hline & GRUPO & N & M edia & Desv. típica \\
\hline \multirow{2}{*}{ I.B.PRETEST } & GCAMA & 4 & 0.17 & 0.04 \\
\cline { 2 - 5 } & GCAME & 5 & 0.26 & 0.05 \\
\hline \multirow{2}{*}{ I.B.TEST } & GCAMA & 5 & 0.25 & 0.05 \\
\cline { 2 - 5 } & GCAME & 4 & 0.27 & 0.07 \\
\hline \multirow{2}{*}{ I.B. POSTEST } & GCAMA & 1 & 0.26 & 0.00 \\
\cline { 2 - 5 } & GCAME & 4 & 0.30 & 0.05 \\
\hline
\end{tabular}


A pesar de que no se han hallado diferencias significativas en los contrastes multivariados, se ha procedido a aplicar la prueba T para el factor inter-sujetos, para lo cual se ha tenido en cuenta que las varianzas de las diferencias entre cada dos niveles de dicho factor fuesen iguales. Esto se comprueba con la prueba de esfericidad de Mauchly, que ha corroborado la hipótesis para cada una de las variables dependientes (0.551) y que, por tanto, nos indica que los datos serán concluyentes.

En la prueba $\mathrm{T}$ de igualdad entre las medias en el factor intersujeto se encuentran diferencias significativas para el IB, ya que el p-valor es inferior a 0.05 (p-valor $=0.016)$. En cambio, esta misma prueba en el test intermedio y postest, no se aprecia la existencia de diferencias significativas en ningún test (tabla 4).

\section{Discusión.}

Después de analizar los resultados nos encontramos que únicamente se han encontrado diferencias significativas en el pretest para el IB entre ambos grupos de entrenamiento (GCAMA y GCAME), habiéndose igualado éste en el test intermedio y postest. El hecho de que se haya igualado la variable IB (relación fuerza velocidad) entre ambos grupos después de seis semanas de tratamiento y que posteriormente, tras cuatro semanas más de entrenamiento esta equiparación se mantenga, nos lleva a pensar que los programas de entrenamientos aplicados han logrado eli-

\begin{tabular}{|c|c|c|}
\hline \multicolumn{3}{|c|}{ Tabla 4. Prueba T para la igualdad de medias en el pretest, test intermedio y } \\
& postest \\
\hline & \multicolumn{2}{|c|}{ Prueba T para la igualdad de medias } \\
\cline { 2 - 3 } & $\mathbf{t}$ & Sig. (bilateral) \\
\hline IB PRETEST & -3.167 & 0.016 \\
\hline $\begin{array}{c}\text { IB TEST } \\
\text { INTERMEDIO }\end{array}$ & -0.515 & 0.622 \\
\hline IB POSTEST & -0.784 & 0.490 \\
\hline
\end{tabular}

minar las diferencias existentes en el IB y que esto es suficiente con seis semanas de entrenamiento, no siendo necesario para conseguir este objetivo un incremento en la duración del tratamiento.

Si comparamos los valores obtenidos del IB en el pretest y postest con otros estudios como es el de Chirosa et al. (2000), podemos observar que las medias de nuestro estudio, son más bajas en todos los casos, siendo el valor más alto obtenido de 0.30 en el postest, mientras que en su estudio, la media más alta del IB alcanza un valor de 0.53 en el postest. Esto puede ser debido a que se han aplicado procedimientos diferentes para su cálculo, como ha sido el SJ100\% en nuestro caso y con el SJ25\% y SJ50\% en el suyo. En este mismo estudio de Chirosa et al. (2000), también podemos observar como se han obtenido resultados significativos para el I.B. en el postest entre grupos, llevándoles a las conclusiones de que el entrenamiento de contraste mejora las distintas manifestaciones de la fuerza dinámica en jóvenes jugadores de balonmano, además de tener un efecto positivo sobre el resto de manifestaciones de fuerza.

Entre las limitaciones que han aparecido durante el desarrollo de esta investigación cabe destacar el elevado número de lesiones que han sufrido las jugadoras, provocadas por el propio lance del juego en competición de máximo nivel, y que han repercutido de un modo directo en el proceso de toma de datos con la consecuente pérdida de información y número de casos que podrían haber arrojado algunos resultados más concluyentes.

\section{Conclusiones.}

Con respecto al objetivo que nos planteamos de mejorar la manifestación de la fuerza menos desarrollada a través del método búlgaro por contraste acentuado (con cargas máximas-pliometría y cargas medias-pliometría) medida por medio del IB, podemos decir que sí se ha logrado una mejora en la manifestación de la fuerza menos desarrollada en los grupos experimentales, por medio de cargas máximas-pliometría para aquellas jugadoras con un índice bajo y con cargas medias-pliometría para aquellas con un índice elevado, consiguiendo iguar el IB entre ambos grupos.

En cambio, cuatro semanas más de entrenamiento no modifican los valores del Índice de Bosco de forma significativa, por lo que prolongar el tratamiento a diez semanas, para equiparar el IB de las jugadoras no tiene ningún sentido.

Finalmente, consideramos que son necesarias más investigaciones con sujetos de elite que permitan aportar más datos al respecto y crear una base sólida de conocimiento científico sobre el que puedan apoyarse monitores y entrenadores a la hora de planificar y seleccionar el método de entrenamiento más adecuado para mejorar las diferentes manifestaciones de la fuerza entre sus jugadores.

\section{Bibliografía.}

Antón, J. L. (1991). Los efectos de un entrenamiento tácticoestratégico individual sobre la optimización del lanzamiento de $7 \mathrm{~m}$. en balonmano en función del análisis de las conductas de interacción en competición. Granada: Tesis Doctoral, Universidad de Granada.

Bosco, C. (1994). La valoración de la fuerza con el test de Bosco. Barcelona: Paidotribo.

Chillón, P., Tercedor, P., Delgado, M. y González, M. (2002). Actividad físico-deportiva en escolares adolescentes. Retos, 3, 5-12.

Chirosa, L. J. (1998). Eficacia del entrenamiento con un método de contraste par la mejora de la fuerza de impulsión en relación a otro tipo convencional en balonmano. Tesis Doctoral, Universidad de Granada, Granada.

Chirosa, L. J., Chirosa, I. y Padial, P. (2000). Efecto del entrenamiento integrado sobre la me mejora de la fuerza de impulsión en un lanzamiento en suspensión en balonmano. Lecturas: Educación Física y Deportes, Revista Digital [en línea] $\mathrm{N}^{\circ}$ 25. Buenos Aires. [Disponible http:// www.efdeportes.com] [Consulta: 2005, 26 de octubre].

Cometti, G. (1988). Bases de la musculación moderna. RED, 6, 2-8.

Cometti, G. (2001). Los métodos modernos de musculación (3 $3^{\mathrm{a}}$ edición). Barcelona: Paidotribo.

García, J. M., Navarro, M. y Ruiz, J.A. (1996). Bases teóricas del entrenamiento deportivo: Principios y Aplicaciones. Madrid: Gymnos.

González, J. J. y Gorostiaga, E. (1995). Fundamentos del entrenamiento de la fuerza. Aplicación al alto rendimiento. Zaragoza: Inde.

Lara, A.J., Abián, J., Alegre, L.M., Jiménez, L. y Aguado, X (2005). Medición directa de la potencia con tests de salto en voleibol femenino. Archivos de Medicina del Deporte, 106, 111-120.

Mouche, M. (2001). Evaluación de la potencia anaeróbica con Ergojump. Lecturas: Educación Física y Deportes, Revista Digital [en línea], № 30. Buenos Aires. [Disponible en: http:/ /www.efdeportes.com] [Consulta: 2005, 15 de octubre].

Nuviala, A., Ruiz, F. y García, M. E. (2003). Tiempo libre, ocio y actividad física en los adolescentes. La influencia de los padres. Retos, 6, 13-20.

Rodríguez, D. y García, J. M. (1998). Efecto de dos modelos de entrenamiento de la fuerza especial en voleibol: aplicación práctica de dos temporadas en el C. V. Gran Canaria. Archivos de Medicina del Deporte, 14, 25-30.

Vittori, C. (1990). El entrenamiento de la fuerza para el sprint. RED, 4, 2-8. 